\title{
Associations of Hemostatic Variables with Cardiovascular Disease and Total Mortality: The Glasgow MONICA Study
}

\author{
Gordon D. O. Lowe ${ }^{1}$ Sanne A. E. Peters ${ }^{2,3,4}$ Ann Rumley ${ }^{1}$ Hugh Tunstall-Pedoe ${ }^{5}$
}

Mark Woodward2,4,50

\footnotetext{
${ }^{1}$ Institute of Cardiovascular and Medical Sciences, University of Glasgow, Glasgow, United Kingdom

2 The George Institute for Global Health, University of New South Wales, Sydney, Australia

3 Julius Center for Health Sciences and Primary Care, University Medical Center Utrecht, Utrecht University, Utrecht, The Netherlands

${ }^{4}$ The George Institute for Global Health, Imperial College London, London, United Kingdom

${ }^{5}$ Cardiovascular Epidemiology Unit, Institute of Cardiovascular Research, University of Dundee, Dundee, United Kingdom
}

\begin{abstract}
Address for correspondence Professor Mark Woodward, PhD, The George Institute for Global Health, Central Working - Fourth Floor, Translation and Innovation Hub, School of Public Health, Imperial College London, 84 Wood Lane, London W12 0BZ, United Kingdom (e-mail: markw@georgeinstitute.org.au).
\end{abstract}

TH Open 2022;6:e107-e113.

\begin{abstract}
Keywords

- hemostasis

- prevention

- cardiovascular disease

- mortality

The associations of plasma levels of hemostatic factors, other than fibrinogen, with risks of cardiovascular disease (CVD) and all-cause mortality are not well defined. In two phases of the Glasgow MONICA study, we assayed coagulation factors (VII, VIII, IX, and von Willebrand factor), coagulation inhibitors (antithrombin, protein C, protein S), coagulation activation markers (prothrombin fragment $1+2$, thrombin-antithrombin complexes, D-dimer), and the fibrinolytic factors, tissue plasminogen activator (t-PA) antigen and plasminogen activator inhibitor type 1 . Over 15 to 20 years, we followed up between 382 and 1,123 men and women aged 30 to 74 years, without baseline CVD, for risks of CVD and mortality. Age- and sex-adjusted hazard ratios (HRs) for CVD (top third vs bottom third) were significant only for factor VIII (1.30; $95 \%$ confidence interval $[\mathrm{Cl}], 1.06-1.58)$ and factor IX $(1.18 ; 95 \% \mathrm{Cl}, 1.01-1.39)$; these HRs were attenuated by further adjustment for CVD risk factors: $1.17(95 \% \mathrm{Cl}, 0.94-1.46)$ and $1.07(95 \% \mathrm{Cl}$, $0.92-1.25)$, respectively. In contrast, factor VIII (HR, 1.63; 95\% Cl, 1.35-1.96), D-dimer ( $\mathrm{HR}, 2.34 ; 95 \% \mathrm{Cl}, 1.26-4.35)$, and t-PA (HR, 2.81; $95 \% \mathrm{Cl}, 1.43-5.54)$ were strongly associated with mortality after full risk factor adjustment. Further studies, including meta-analyses, are required to assess the associations of these hemostatic factors with the risks of stroke and heart disease and causes of mortality.
\end{abstract}

\section{Introduction}

Plasma levels of some hemostatic factors and activation markers have been associated with the risk of arterial cardiovascular diseases (CVDs): coronary heart disease

received

December 13, 2021

accepted after revision

February 28, 2022
DOI https://doi.org/

10.1055/a-1789-4896.

ISSN 2512-9465.
(CHD), stroke, and peripheral arterial disease (PAD). ${ }^{1,2} \mathrm{Fi}-$ brinogen is associated with risks of CHD, stroke, and total mortality ${ }^{3}$ and may add to risk prediction scores for CVD risk. ${ }^{4,5} \mathrm{~A}$ meta-analysis has established significant, but (c) 2022. The Author(s).

This is an open access article published by Thieme under the terms of the Creative Commons Attribution License, permitting unrestricted use, distribution, and reproduction so long as the original work is properly cited. (https://creativecommons.org/licenses/by/4.0/)

Georg Thieme Verlag KG, Rüdigerstraße 14, 70469 Stuttgart, Germany 
weaker, associations of plasma levels of von Willebrand factor (VWF), tissue plasminogen activator (t-PA) antigen, and the hemostatic activation marker fibrin D-dimer with the risk of CHD. ${ }^{6}$ There is increasing evidence that these three markers are also associated with risks of stroke ${ }^{7}$ and PAD. ${ }^{8}$

For other hemostatic factors, inhibitors, and activation markers, there are insufficient data to assess with confidence their associations with CVD or mortality. ${ }^{2}$ From the prospective Glasgow MONICA (Monitoring of Trends and Determinants of Cardiovascular Disease) study, we now report the associations with incident CVD (CHD and stroke), and with total mortality, of plasma levels of coagulation factors VII, VIII, and IX; coagulation activation markers prothrombin fragment $1+2(\mathrm{~F} 1+2)$, thrombin-antithrombin (TAT) complexes, and D-dimer; coagulation inhibitors antithrombin and protein $\mathrm{C}$ and protein $\mathrm{S}$; activated partial thromboplastin time (aPTT), activated protein C (APC) resistance, and its common genetic determinant (the FV:R506Q mutation); and the fibrinolytic variables t-PA antigen and plasminogen activator inhibitor type 1 (PAI-1) activity.

\section{Methods}

\section{Study Participants}

The Glasgow MONICA study recruited random population samples of men and women aged 25 to 64 years in four phases: 1984-1987 (as part of the Scottish Heart Health Study); 1989; 1992 (aged 25-74 years); and 1995. ${ }^{9}$ Data were subsequently combined to form the Scottish Heart Health Extended Cohort Study (SHHEC), from which a cardiovascular risk assessment score (the ASSIGN score) was developed for use in National Health Service Scotland. ${ }^{10}$ All participants completed a questionnaire, which solicited information on demographics, past medical history, and lifestyle, including tobacco use. They were invited to attend clinics where blood pressure, weight, and height were measured, a 12-lead electrocardiogram (ECG) was applied, and a blood sample taken. The present study was restricted to those aged 30 to 74 years, free from clinical evidence of CVD at baseline, and having data on at least one hemostatic variable other than fibrinogen. CVD was diagnosed if they reported having received a previous doctor diagnosis of angina, heart attack, or stroke or previously undergoing coronary artery bypass graft (CABG) or percutaneous transluminal coronary angioplasty (PTCA) on the baseline questionnaire; if the ECG was suggestive of myocardial infarction (using Minnesota codes); or if the national records obtained showed that they had been hospitalized for CHD or stroke or had undergone any coronary surgical procedures prior to recruitment (and post-1980).

\section{Follow-up and End Point Definition}

Individuals who gave written permission were followed up for cause-specific mortality through linkage with two national systems: death registrations and the record linkage database. ${ }^{10}$ The latter was also used to follow up, and trace back, hospitalizations. Records were obtained from 1981 to 2009. ${ }^{4}$ CVD end points were: deaths attributed to a cardio- vascular cause (International Classification of Disease [ICD] 9 codes 390-459, ICD 10 codes 100-199); any hospital discharge diagnosis postrecruitment of CHD (ICD 9 codes 410414 , ICD 10 codes120-125) or cerebrovascular disease (ICD 9 codes 430-438, ICD 10 G45, 160-169); or surgical codes for CABG or PTCA.

\section{Hemostatic Variables}

Blood was anticoagulated with $0.11 \mathrm{~mol} / \mathrm{L}$ trisodium citrate $(9: 1 \mathrm{v} / \mathrm{v})$ and centrifuged, and aliquots were stored at $-80^{\circ} \mathrm{C}$. Four hemostatic variables other than fibrinogen were studied in the 1989 survey. ${ }^{11}$ VWF was assayed using an in-house enzyme-linked immunoassay (ELISA) employing rabbit antihuman polyclonal antibodies, obtained from DAKO plc, High Wycombe, United Kingdom. t-PA antigen was measured with a commercial ELISA (Biopool AB, Umea, Sweden). PAI-1 activity was measured with a commercial kit (Kabi, Sweden). D-dimer was assayed by a commercial ELISA (Dimertest; Agen Biomedical, Parsippany, New Jersey, United States). Significant baseline correlations with demographic and cardiovascular risk factor variables on multivariable analyses for these variables were age and triglycerides. ${ }^{11}$

Ten other hemostatic variables were studied in the 1992 survey: coagulation factors VII, VIII, and IX, antithrombin and protein $\mathrm{C}$ and protein $\mathrm{S}$ activities, $\mathrm{F} 1+2$, TAT, aPTT, APC resistance, and the FV:R506Q mutation. Their assays, distributions, and correlations with baseline demographic and cardiovascular risk factor variables have been described. ${ }^{12-14}$

\section{Statistical Methods}

Data from the 1989 and 1992 MONICA cohorts were analyzed as two separate cohorts, reflecting the fact that different hemostatic variables were measured in the two baseline surveys. Cox proportional hazards models were used to estimate the hazard ratios (HRs) and 95\% confidence intervals (Cls) for CVD, and total mortality, for each hemostatic variable. We predefined two models: model 1 was adjusted for age and sex only; model 2 was adjusted for the CVD risk factors also in the ASSIGN score: systolic blood pressure, serum total and highdensity lipoprotein cholesterol, cigarettes smoked per day, diabetes status, family history of CHD, and the Scottish Index of Multiple Deprivation (SIMD) score. Analyses were conducted for a 1 standard deviation (SD) increase in level of each hemostatic variable; and for the highest third compared with the lowest third of its distribution. All statistical analyses were performed using R, version 2.15. 3 (R Foundation for Statistical Computing, Vienna, Austria).

\section{Results}

\section{Survey: PAI-1, VWF, t-PA, D-dimer}

- Table 1 shows the baseline characteristics of participants in the second phase of the Glasgow MONICA study in 1989: baseline levels of PAI-1, VWF, D-dimer, and t-PA; and outcomes. During a median (interquartile interval) of 20.6 (20.5-20.8) years, 181 CVD events and 173 deaths occurred. Of 706 participants, citrated plasma aliquots were available, after routine measurement of plasma 
Table 1 Baseline summary statistics: 1989 MONICA survey

\begin{tabular}{|l|l|l|}
\hline Variable & \multicolumn{2}{l|}{$n$} \\
\hline Cardiovascular risk factors & 706 & $48(10)$ \\
\hline Age (y), mean (SD) & 353 & 50 \\
\hline Male sex, \% & 706 & $132(21)$ \\
\hline Systolic blood pressure (mm Hg), mean (SD) & 706 & $5.9(1.2)$ \\
\hline Total cholesterol (mmol/L), mean (SD) & 706 & $1.2(0.3)$ \\
\hline HDL cholesterol (mmol/L), mean (SD) & $26(4)$ \\
\hline Body mass index (kg/m²), mean (SD) & 705 & 43 \\
\hline Current smokers, \% & 706 & 3 \\
\hline Diabetes, \% & 706 & 26 \\
\hline Family history of CVD, \% & 706 & $45(24)$ \\
\hline SIMD, median (IQI), mean (SD) & 706 & \\
\hline Hemostatic variables & & \\
\hline PAl-1 activity (AU/mL), mean (SD) & 523 & $98.5(46.5)$ \\
\hline VWF (IU/dL), median (IQI) & 405 & $91(73-118)$ \\
\hline D-dimer (ng/mL), median (IQI) & 384 & $56(37-78)$ \\
\hline t-PA antigen (ng/mL), median (IQI) & 382 & $3.9(5.5-8.5)$ \\
\hline
\end{tabular}

Abbreviations: CVD, cardiovascular disease; HDL, high-density lipoprotein; IQI, interquartile interval; PAI-1, plasminogen activator inhibitor type 1; SD, standard deviation; t-PA, tissue plasminogen activator; VWF, von Willebrand factor.

Note: Decreasing number of available samples reflects decreasing availability of plasma aliquot.

fibrinogen, from 523 participants for assay of PAI-1. Thereafter, as laboratory assays were developed sequentially for VWF, D-dimer, and t-PA antigens, residual plasma aliquots were sufficient for assay in 405, 384, and 382 participants, respectively.y.

- Table 2 shows the associations of these hemostatic variables with the study outcomes, both as a 1 SD increase in levels and as ratio of top third to bottom third of levels. PAI-1 and VWF showed no association with CVD or mortality after full adjustment for ASSIGN variables. D-dimer and t-PA showed associations with CVD and mortality after full adjustment-HRs, top third versus bottom third: 2.34 (95\% CI, 1.264.35) and 2.81 (95\% CI, 1.43-5.54); and per 1 SD increase: 1.16 (95\% CI, 0.99-1.36) and 1.63 (95\% CI, 1.35-1.96).

Table 2 Associations with outcomes: 1989 MONICA survey

\begin{tabular}{|c|c|c|c|c|}
\hline & \multicolumn{2}{|l|}{ CVD } & \multicolumn{2}{|l|}{ Total mortality } \\
\hline & \multicolumn{2}{|l|}{ Adjustments } & \multicolumn{2}{|l|}{ Adjustments } \\
\hline Variable & Age and sex & ASSIGN & Age and sex & ASSIGN \\
\hline \multicolumn{5}{|c|}{ HRs for a 1 SD increase in level } \\
\hline PAI-1 & $1.18(1.01-1.39)$ & $1.07(0.92-1.25)$ & $1.12(0.95-1.32)$ & $1.06(0.90-1.25)$ \\
\hline VWF & $1.02(0.85-1.23)$ & $0.99(0.83-1.18)$ & $1.05(0.88-1.25)$ & $1.01(0.84-1.20)$ \\
\hline D-dimer & $1.18(1.01-1.37)$ & $1.17(1.00-1.38)$ & $1.18(1.02-1.38)$ & $1.16(0.99-1.36)$ \\
\hline $\mathrm{t}-\mathrm{PA}$ & $1.30(1.06-1.58)$ & $1.17(0.94-1.46)$ & $1.62(1.36-1.92)$ & $1.63(1.35-1.96)$ \\
\hline \multicolumn{5}{|c|}{ HRs for top third vs bottom third of distribution } \\
\hline PAI-1 & $1.08(0.72-1.62)$ & $0.95(0.63-1.44)$ & $1.20(0.78-1.86)$ & $1.11(0.71-1.74)$ \\
\hline VWF & $1.01(0.62-1.65)$ & $1.03(0.63-1.69)$ & $1.10(0.67-1.80)$ & $1.01(0.61-1.66)$ \\
\hline D-dimer & $1.28(0.77-2.13)$ & $1.39(0.83-2.32)$ & $2.26(1.23-4.15)$ & $2.34(1.26-4.35)$ \\
\hline $\mathrm{t}-\mathrm{PA}$ & $1.49(0.84-2.63)$ & $1.09(0.61-1.97)$ & $3.20(1.64-6.24)$ & $2.81(1.43-5.54)$ \\
\hline
\end{tabular}

Abbreviations: CVD, cardiovascular disease; HRs, hazard ratios; PAI-1, plasminogen activator inhibitor type 1; SD, standard deviation; t-PA, tissue plasminogen activator; VWF, von Willebrand factor.

Note: HRs and 95\% confidence intervals for CVD and total mortality. Analyzed first for a 1 SD increase in hemostatic factor level and then for top third versus bottom third of distribution of level. Adjusted for age and sex and then also for ASSIGN risk variables (systolic blood pressure, serum total and high-density lipoprotein cholesterol, cigarettes smoked per day, diabetes status, family history of coronary heart disease, and the Scottish Index of Multiple Deprivation [SIMD] score). 
1992 Survey: Factors VII, VIII, and IX, Antithrombin, Protein C, Protein S, F1 + 2, TAT, aPTT, APC Resistance, FV:R506Q Mutation

- Table 3 shows the baseline characteristics of participants in the third phase of the Glasgow MONICA study in 1992; baseline levels of coagulation factors, inhibitors, and activation markers; and outcomes. During a median (interquartile interval) of 17.5 (15.4-17.8) years, 381 CVD events and 401 deaths occurred. Of 1,324 participants, citrated plasma aliquots were available, after routine measurement of plasma fibrinogen, from 1,088 to 1,123 participants for assay of factors VII, VIII, IX, antithrombin, and protein C activities. Thereafter, as laboratory assays were developed sequentially for protein $\mathrm{S}, \mathrm{F} 1+2$, and TAT antigens, residual plasma aliquots were sufficient for assay in 843 to 896 participants and then for aPTT and APC ratio in 709 to 715 participants.

- Table 4 shows the associations of these hemostatic variables with the study outcomes, both as a 1 SD increase in levels and as ratio of top third to bottom third of levels. Age- and sex-adjusted HRs for CVD, top third versus bottom third, were significant only for factor VIII $(1.30$; $95 \%$ CI, 1.06-
$1.58)$ and factor IX (1.18; $95 \% \mathrm{CI}, 1.01-1.39)$; these HRs were attenuated by further adjustment for CVD risk factors, respectively, to 1.17 (95\% CI, 0.94-1.46) and 1.07 (95\% CI, 0.921.25). In contrast, factor VIII was strongly associated with total mortality after full risk factor adjustment: HRs, top third versus bottom third, 1.63 (95\% CI, 1.35-1.96) and per 1 SD increase 1.14 (95\% CI, 1.03-1.28). No other hemostatic variable measured in this survey was significantly associated with either CVD or with total mortality.

\section{Discussion}

In this study of the associations of plasma levels of 15 hemostatic variables with risks of CVD, and of total mortality, in two random population samples of men and women aged 30 to 74 years, age- and sex-adjusted HRs for CVD were significant for factor VIII, factor IX, D-dimer, and t-PA. Apart from D-dimer, these were attenuated by further adjustment for CVD risk factors. In contrast, factor VIII, D-dimer, and t-PA were strongly associated with total mortality after full risk factor adjustment.

Table 3 Baseline summary statistics: 1992 MONICA survey

\begin{tabular}{|c|c|c|}
\hline Variable & $n$ & \\
\hline \multicolumn{3}{|l|}{ Cardiovascular risk factors } \\
\hline Age $(y)$, mean (SD) & 1,324 & $51(13)$ \\
\hline Male sex, $\%$ & 1,324 & 47 \\
\hline Systolic blood pressure (mm Hg), mean (SD) & 1,324 & $133(24)$ \\
\hline Total cholesterol (mmol/L), mean (SD) & 1,324 & $6.1(1.2)$ \\
\hline HDL cholesterol (mmol/L), mean (SD) & 1,324 & $1.5(0.4)$ \\
\hline Body mass index $\left(\mathrm{kg} / \mathrm{m}^{2}\right)$, mean (SD) & 1,313 & $26(5)$ \\
\hline Current smokers, $\%$ & 1,324 & 42 \\
\hline Diabetes, \% & 1,324 & 1 \\
\hline Family history of CVD, \% & 1,324 & 28 \\
\hline SIMD, median (IQI), mean (SD) & 1,324 & $46(24)$ \\
\hline \multicolumn{3}{|l|}{ Hemostatic variables } \\
\hline Factor VII (IU/dL), mean (SD) & 1,115 & $109.9(24.3)$ \\
\hline Factor VIII (IU/dL), mean (SD) & 1,121 & $146.3(50.0)$ \\
\hline Factor IX (IU/dL), mean (SD) & 1,113 & $131.4(33.8)$ \\
\hline Antithrombin (IU/dL), mean (SD) & 1,088 & $100.2(12.9)$ \\
\hline Protein C (IU/dL), mean (SD) & 1,123 & $108.6(31.9)$ \\
\hline Protein S (\%pool), mean (SD) & 896 & $130.7(29.4)$ \\
\hline $\mathrm{F} 1+2(\mathrm{ng} / \mathrm{mL})$, median (IQI) & 887 & $2.12(1.53-2.95)$ \\
\hline TAT (ng/mL), median (IQI) & 843 & $4.09(2.73-7.36)$ \\
\hline aPTT (s), mean (SD) & 709 & $31.1(4.3)$ \\
\hline APC ratio, mean (SD) & 715 & $2.86(0.54)$ \\
\hline Heterozygous FV:R506Q (\%) & 1293 & 2.5 \\
\hline
\end{tabular}

Abbreviations: APC, activated protein C; aPTT, activated partial thromboplastin time; CVD, cardiovascular disease; F1 + 2, prothrombin fragment $1+2$; HDL, high-density lipoprotein; IQI, interquartile interval; SD, standard deviation; TAT, thrombin-antithrombin.

Note: Decreasing number of available samples reflects decreasing availability of plasma aliquots. 
Table 4 Associations with outcomes: 1992 MONICA survey

\begin{tabular}{|c|c|c|c|c|}
\hline & \multicolumn{2}{|l|}{ CVD } & \multicolumn{2}{|l|}{ Total mortality } \\
\hline & \multicolumn{2}{|l|}{ Adjustments } & \multicolumn{2}{|l|}{ Adjustments } \\
\hline & Age and sex & ASSIGN score & Age and sex & ASSIGN score \\
\hline \multicolumn{5}{|c|}{ HRs for a 1 SD increase in level $\left({ }^{*} \log\right.$ transformed) } \\
\hline Factor VII & $1.12(1.00-1.26)$ & $1.07(0.95-1.21)$ & $1.09(0.97-1.22)$ & $1.09(0.97-1.23)$ \\
\hline Factor VIII & $1.10(0.99-1.22)$ & $1.09(0.97-1.22)$ & $1.13(1.02-1.26)$ & $1.14(1.03-1.28)$ \\
\hline Factor IX & $1.10(0.98-1.22)$ & $1.02(0.91-1.15)$ & $1.09(0.98-1.21)$ & $1.06(0.94-1.18)$ \\
\hline Antithrombin & $1.06(0.95-1.18)$ & $1.10(0.89-1.12)$ & $1.02(0.92-1.14)$ & $1.00(0.89-1.11)$ \\
\hline Protein C & $0.94(0.84-1.05)$ & $0.92(0.82-1.04)$ & $0.90(0.81-1.01)$ & $0.91(0.81-1.02)$ \\
\hline Protein S & $1.14(1.00-1.30)$ & $1.06(0.93-1.20)$ & $1.08(0.95-1.23)$ & $1.03(0.90-1.17)$ \\
\hline $\mathrm{F} 1+2^{*}$ & $1.12(0.99-1.27)$ & $1.09(0.96-1.25)$ & $1.02(0.90-1.15)$ & $1.02(0.90-1.16)$ \\
\hline TAT* & $1.04(0.91-1.19)$ & $1.02(0.89-1.17)$ & $0.96(0.85-1.09)$ & $0.94(0.82-1.07)$ \\
\hline aPTT & $0.94(0.81-1.09)$ & $0.92(0.79-1.08)$ & $1.03(0.90-1.18)$ & $1.03(0.89-1.19)$ \\
\hline APC ratio & $0.97(0.83-1.13)$ & $1.00(0.86-1.16)$ & $0.98(0.84-1,14)$ & $0.98(0.84-1.14)$ \\
\hline \multicolumn{5}{|c|}{ HRs for top third versus bottom third of distribution } \\
\hline Factor VII & $1.02(0.85-1.23)$ & $0.99(0.83-1.18)$ & $1.05(0.88-1.25)$ & $1.01(0.84-1.20)$ \\
\hline Factor VIII & $1.30(1.06-1.58)$ & $1.17(0.94-1.46)$ & $1.62(1.36-1.92)$ & $1.63(1.35-1.96)$ \\
\hline Factor IX & $1.18(1.01-1.39)$ & $1.07(0.92-1.25)$ & $1.12(0.95-1.32)$ & $1.06(0.90-1.25)$ \\
\hline Antithrombin & $1.25(0.95-1.64)$ & $1.11(0.84-1.47)$ & $1.19(0.91-1.55)$ & $1.12(0.85-1.48)$ \\
\hline Protein C & $0.84(0.64-1.10)$ & $0.82(0.62-1.08)$ & $0.82(0.63-1.06)$ & $0.84(0.64-1.10)$ \\
\hline Protein S & $1.18(0.86-1.62)$ & $1.03(0.75-1.41)$ & $1.17(0.85-1.60)$ & $1.04(0.75-1.43)$ \\
\hline $\mathrm{F} 1+2$ & $1.37(0.99-1.89)$ & $1.23(0.89-1.71)$ & $1.15(0.84-1.58)$ & $1.11(0.81-1.52)$ \\
\hline TAT & $0.96(0.69-1.33)$ & $0.94(0.68-1.31)$ & $0.86(0.63-1.18)$ & $0.81(0.59-1.12)$ \\
\hline aPTT & $1.06(0.74-1.52)$ & $0.98(0.68-1.41)$ & $1.17(0.82-1.66)$ & $1.16(0.81-1.66)$ \\
\hline APC ratio & $0.94(0.65-1.35)$ & $1.03(0.70-1.49)$ & $0.95(0.67-1,35)$ & $0.99(0.69-1.42)$ \\
\hline
\end{tabular}

Abbreviations: APC, activated protein C; aPTT, activated partial thromboplastin time; CVD, cardiovascular disease; F1 + 2, prothrombin fragment $1+2$; HRs, hazard ratios; SD, standard deviation; TAT, thrombin-antithrombin.

Note: HRs and 95\% confidence intervals for CVD and total mortality. Analyzed first for a 1 SD increase in hemostatic factor level and then for top third versus bottom third of distribution of level. Adjusted for age and sex and then also for ASSIGN risk variables (systolic blood pressure, serum total and high-density lipoprotein cholesterol, cigarettes smoked per day, diabetes status, family history of coronary heart disease, and the Scottish Index of Multiple Deprivation [SIMD] score). Log transformation was required for variable F1+2 and TAT, for calculation of HRs for a 1SD increase in level (top half of - Table 4 ).

Factor VII level was not associated with CVD or mortality in our study after risk factor adjustment. This is consistent with recent reviews of studies ${ }^{2,15}$ and with meta-analyses of functional mutations in the factor VII gene, which showed no associations with CHD. ${ }^{15,16}$

The association of factor VIII level with CVD (HR, top to bottom third, 1.30; 95\% CI, 1.06-1.58) was attenuated after risk factor adjustment. There is uncertainty in the literature on this association. ${ }^{2,17-26}$ We observed no significant association of its carrier protein, VWF, with CVD; a meta-analysis of the association of VWF level with CHD in 15 studies with 6,556 cases showed a significant association: HR per $1 \mathrm{SD}$, 1.16 (95\% CI, 1.10-1.22). ${ }^{6}$ Plasma VWF, as its carrier protein, is correlated with plasma factor VIII. ${ }^{17,18}$

Plasma factor VIII was associated with total mortality in our study, after risk factor adjustment (HR, 1.63; 95\% CI, 1.35-1.96). This association has also been reported from the
Multi-Ethnic Study of Atherosclerosis ${ }^{19}$ and the MEGA study, ${ }^{20}$ which suggested that environmental factors, such as chronic comorbidities and chronic inflammation, are at least in part responsible. While we did not observe an association of VWF with mortality in the present study, this association was found in the MEGA study. ${ }^{20}$

Factor IX level was weakly associated with CVD after adjustment for age and sex (HR, top to bottom third, 1.18; 95\% CI, 1.01-1.39) and attenuated after further adjustment for risk factors in the ASSIGN score (HR, 1.07; 95\% CI, 0.921.25). As with factor VIII, there is uncertainty in the literature on this association. ${ }^{2,27,28}$ We did not observe an association of factor IX with mortality.

Among coagulation activation markers, $\mathrm{F} 1+2$ showed a borderline association with CHD when adjusted for age and sex, but no coagulation activation marker was associated with incident CVD after additional adjustment for risk 
factors. Studies of the associations of F1 +2 and TAT complexes with risk of CVD are inconclusive. ${ }^{21-23} \mathrm{~A}$ meta-analysis found a significant association of D-dimer levels with CHD: HR, 1.23 (95\% CI, 1.16-1.32; 18 studies with 6,799 (ases). ${ }^{6}$

Among coagulation inhibitors, protein $\mathrm{S}$ showed a borderline association with CHD when adjusted for age and sex; but no coagulation inhibitor was associated with incident CVD after additional adjustment for age, sex, and risk factors in the ASSIGN score; nor with total mortality. The Atherosclerosis Risk in Communities (ARIC) study found a similar lack of association for antithrombin and protein $\mathrm{C}$ for $\mathrm{CHD}^{24,25}$ but found an increased risk of stroke for low protein $C$ in longerterm follow-up. ${ }^{25,26} \mathrm{~A}$ meta-analysis confirmed increased risk of stroke for deficiencies of proteins $\mathrm{C}$ and $\mathrm{S}$, but not of antithrombin. ${ }^{29}$ No increase in mortality has been reported in persons with deficiencies of coagulation inhibitors. ${ }^{30}$

aPTT was not associated with CHD in our study, as in the ARIC study. ${ }^{26,27}$ Neither APC resistance nor its major genetic determinant, the FV:R506Q mutation, was associated with CHD in our study; however, meta-analyses have shown significant associations of $\mathrm{FV}$ :R506Q with $\mathrm{CHD}^{16}$ and stroke. $^{29}$ We observed a borderline association of FV: R506Q with total mortality, but this was not observed in a larger study. ${ }^{30}$

The fibrinolytic variables, tPA antigen and PAI-1 activity, did not show significant adjusted associations with incident CVD in our study. The association of tPA was, however, consistent with a meta-analysis of incident CHD (HR, 1.13; $95 \% \mathrm{CI}, 1.06-1.21$; 13 studies with 5,494 cases). ${ }^{6}$ The association of PAI-1 with incident CVD was consistent with that in another meta-analysis of its associations with CHD risk, which reported an HR for top third to bottom third of 0.98 (95\% CI, 0.53-1.87). ${ }^{31}$

In contrast, t-PA was significantly associated with total mortality after adjustment for age, sex, and risk factors in the ASSIGN score (HR, top third to bottom third, 2.81; $95 \% \mathrm{CI}$, 1.43-5.54). This association was also observed in the PROSPER study cohort. ${ }^{23}$

Strengths of our study include that it is a large random sample of a general population of men and women aged 30 to 74 years, followed for up to 20 years for both CVD and total mortality. Also, the study assessed 15 hemostatic variables in a combined analysis, using well-calibrated laboratory assays, applying national or international standards, where available. ${ }^{12}$ We had a limited number of outcome events, which precluded reliable estimation of associations with stroke and CHD separately or of causes of mortality. We were also unable to look at changes in hemostatic variables over time, having only baseline values. Another limitation was the largely Scottish ethnic population, and hence results may not be generalizable to other populations, worldwide.

\section{Conclusion}

In this study, we found no compelling evidence of independent associations of hemostatic variables with CVD, suggesting that they may not add significantly to CVD risk stratification. We report that factor VIII, D-dimer, and t-PA were strongly associated with total mortality after full risk factor adjustment, but found no such association for other hemostatic variables. Further studies and meta-analyses are required to reliably assess the associations of the hemostatic variables we have studied with the risks of components of CVD, particularly stroke and CHD, ${ }^{6}$ and causes of mortality.

\section{What Is Known about This Topic?}

- Apart from fibrinogen, there are limited data on the associations of hemostatic factors with risks of CVD and total mortality.

\section{What Does This Paper Add?}

- Of 15 hemostatic variables assayed in a well-defined cohort followed for 17 years, factors VIII and IX were associated with risks of CVD after adjustment for age and sex; but these associations were attenuated after adjustment for CVD risk factors.

- Factor VIII, D-dimer, and t-PA were associated with total mortality after adjustment for age, sex, and CVD risk factors.

\section{Authors' Contributions}

G. D. O. L., A. R., M. W., and H. T.-P. performed the study of hemostatic factors in the Glasgow MONICA study. S. A. E. P. and M. W. performed the analyses of follow-up data. G. D. O. L. drafted the manuscript, and all co-authors provided critical review.

\section{Funding}

This study was funded by the Chief Scientist Office, Department of Health, Scottish Office; the views expressed in this report are those of the authors and not necessarily of this office.

\section{Conflict of Interest}

M. W. is a consultant to Amgen, Kyowa, Kirin, and Freeline. G. D. O. L. is a consultant to Freeline. S. A. E. P., A. R., and H. T.-P. declare no conflicts of interest.

\section{References}

1 Lowe GDO. Can haematological tests predict cardiovascular risk? The 2005 Kettle Lecture. Br J Haematol 2006;133(03):232-250

2 Lowe G, Rumley A. The relevance of coagulation in cardiovascular disease: what do the biomarkers tell us? Thromb Haemost 2014; 112(05):860-867

3 Danesh J, Lewington S, Thompson SG, et al; Fibrinogen Studies Collaboration. Plasma fibrinogen level and the risk of major cardiovascular diseases and nonvascular mortality: an individual participant meta-analysis. JAMA 2005;294(14):1799-1809

4 Woodward M, Tunstall-Pedoe H, Rumley A, Lowe GDO. Does fibrinogen add to prediction of cardiovascular disease? Results from the Scottish Heart Health Extended Cohort Study. Br J Haematol 2009;146(04):442-446 
5 Kaptoge S, Di Angelantonio E, Pennells L, et al; Emerging Risk Factors Collaboration. C-reactive protein, fibrinogen, and cardiovascular disease prediction. N Engl J Med 2012;367(14): 1310-1320

6 Willeit P, Thompson A, Aspelund T, et al. Hemostatic factors and risk of coronary heart disease in general populations: new prospective study and updated meta-analyses. PLoS One 2013;8 (02):e55175

7 Wannamethee SG, Whincup PH, Lennon L, Rumley A, Lowe GD. Fibrin D-dimer, tissue-type plasminogen activator, von Willebrand factor, and risk of incident stroke in older men. Stroke 2012;43(05):1206-1211

8 Tzoulaki I, Murray GD, Lee AJ, Rumley A, Lowe GDO, Fowkes FGR. Inflammatory, haemostatic, and rheological markers for incident peripheral arterial disease: Edinburgh Artery Study. Eur Heart J 2007;28(03):354-362

9 Tunstall-Pedoe H, Kuulasmaa K, Tolonen H, et al. MONICA Monograph and Multimedia Sourcebook: World's Largest Study of Heart Disease, Stroke, Risk Factors, and Population Trends 1979-2002. Geneva: World Health Organization; 2003

10 Woodward M, Brindle P, Tunstall-Pedoe H. SIGN group on risk estimation. Adding social deprivation and family history to cardiovascular risk assessment: the ASSIGN score from the Scottish Heart Health Extended Cohort (SHHEC). Heart 2007;93(02):172-176

11 Rumley A. Fibrinolytic and Endothelial Markers in Cardiovascular Disease and Diabetes Mellitus [PhD Thesis]. Glasgow: University of Glasgow; 1996

12 Lowe GDO, Rumley A, Woodward M, et al. Epidemiology of coagulation factors, inhibitors and activation markers: the Third Glasgow MONICA Survey. I. Illustrative reference ranges by age, sex and hormone use. Br J Haematol 1997;97(04):775-784

13 Woodward M, Lowe GDO, Rumley A, et al. Epidemiology of coagulation factors, inhibitors and activation markers: The Third Glasgow MONICA Survey. II. Relationships to cardiovascular risk factors and prevalent cardiovascular disease. Br J Haematol 1997; 97(04):785-797

14 Lowe GDO, Rumley A, Woodward M, Reid E, Rumley J. Activated protein $\mathrm{C}$ resistance and the FV:R506Q mutation in a random population sample-associations with cardiovascular risk factors and coagulation variables. Thromb Haemost 1999;81(06): 918-924

15 Bernardi F, Mariani G. Biochemical, molecular and clinical aspects of coagulation factor VII and its role in hemostasis and thrombosis. Haematologica 2021;106(02):351-362

16 Ye Z, Liu EHC, Higgins JPT, et al. Seven haemostatic gene polymorphisms in coronary disease: meta-analysis of 66,155 cases and 91,307 controls. Lancet 2006;367(9511):651-658

17 Rumley A, Lowe GDO, Sweetnam PM, Yarnell JWG, Ford RP. Factor VIII, von Willebrand factor and the risk of major ischaemic heart disease in the Caerphilly Heart Study. Br J Haematol 1999;105 (01):110-116
18 Rietveld IM, Lijfering WM, le Cessie S, et al. High levels of coagulation factors and venous thrombosis risk: strongest association for factor VIII and von Willebrand factor. J Thromb Haemost 2019;17(01):99-109

19 Folsom AR, Delaney JAC, Lutsey PL, et al; Multiethnic Study of Atherosclerosis Investigators. Associations of factor VIIIc, D-dimer, and plasmin-antiplasmin with incident cardiovascular disease and all-cause mortality. Am J Hematol 2009;84(06):349-353

20 Yap ES, Timp JF, Flinterman LE, et al. Elevated levels of factor VIII and subsequent risk of all-cause mortality: results from the MEGA follow-up study. J Thromb Haemost 2015;13(10):1833-1842

21 Cooper JA, Miller GJ, Bauer KA, et al. Comparison of novel hemostatic factors and conventional risk factors for prediction of coronary heart disease. Circulation 2000;102(23):2816-2822

22 Smith A, Patterson C, Yarnell J, Rumley A, Ben-Shlomo Y, Lowe G. Which hemostatic markers add to the predictive value of conventional risk factors for coronary heart disease and ischemic stroke? The Caerphilly Study. Circulation 2005;112(20):3080-3087

23 Rumley A, Lowe G, Stott D, et al. Coagulation activation markers are associated with cardiovascular events and death in the elderly at risk: PROSPER study cohort. J Thromb Haemost 2009;7 (Suppl 2):Abstract OC-WE-33

24 Folsom AR, Wu KK, Rosamond WD, Sharrett AR, Chambless LE. Prospective study of hemostatic factors and incidence of coronary heart disease: the Atherosclerosis Risk in Communities (ARIC) Study. Circulation 1997;96(04):1102-1108

25 Folsom AR, Ohira T, Yamagishi K, Cushman M. Low protein C and incidence of ischemic stroke and coronary heart disease: the Atherosclerosis Risk in Communities (ARIC) Study. J Thromb Haemost 2009;7(11):1774-1778

26 Folsom AR, Rosamond WD, Shahar E, et al; The Atherosclerosis Risk in Communities (ARIC) Study Investigators. Prospective study of markers of hemostatic function with risk of ischemic stroke. Circulation 1999;100(07):736-742

27 Yamagishi K, Aleksic N, Hannan PJ, Folsom ARARIC Study Inverstigators. Coagulation factors II, V, IX, X, XI, and XII, plasminogen, and alpha-2 antiplasmin and risk of coronary heart disease. J Atheroscler Thromb 2010;17(04):402-409

28 Olson NC, Cushman M, Judd SE, et al. Associations of coagulation factors IX and XI levels with incident coronary heart disease and ischemic stroke: the REGARDS study. J Thromb Haemost 2017;15 (06):1086-1094

29 Chiasakul T, De Jesus E, Tong J, et al. Inherited thrombophilia and the risk of arterial ischemic stroke: a systematic review and metaanalysis. J Am Heart Assoc 2019;8(19):e012877

30 Pabinger I, Vossen CY, Lang J, et al. Mortality and inherited thrombophilia: results from the European Prospective Cohort on Thrombophilia. J Thromb Haemost 2012;10(02):217-222

31 Lowe GDO, Danesh J, Lewington S, et al. Tissue plasminogen activator antigen and coronary heart disease. Prospective study and meta-analysis. Eur Heart J 2004;25(03):252-259 